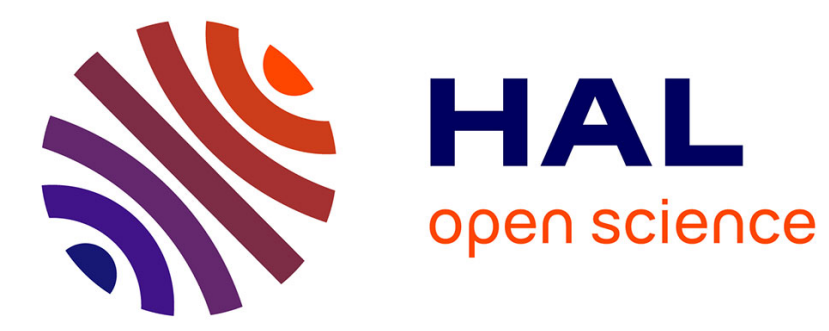

\title{
Stability of the Kalman filter for continuous time output error systems
}

Boyi Ni, Qinghua Zhang

\section{To cite this version:}

Boyi Ni, Qinghua Zhang. Stability of the Kalman filter for continuous time output error systems. Systems and Control Letters, 2016, 94, pp.172 - 180. 10.1016/j.sysconle.2016.06.006 . hal-01376819

\section{HAL Id: hal-01376819 \\ https://inria.hal.science/hal-01376819}

Submitted on 5 Oct 2016

HAL is a multi-disciplinary open access archive for the deposit and dissemination of scientific research documents, whether they are published or not. The documents may come from teaching and research institutions in France or abroad, or from public or private research centers.
L'archive ouverte pluridisciplinaire $\mathbf{H A L}$, est destinée au dépôt et à la diffusion de documents scientifiques de niveau recherche, publiés ou non, émanant des établissements d'enseignement et de recherche français ou étrangers, des laboratoires publics ou privés. 


\title{
Stability of the Kalman Filter for Continuous Time Output Error Systems
}

\author{
Boyi $\mathrm{Ni}^{\mathrm{a}}$, Qinghua Zhang ${ }^{\mathrm{b}}$ \\ ${ }^{a}$ SAP Labs China, 1001 Chenhui Rd., Shanghai 201203, China (previously with Inria) \\ ${ }^{b}$ Inria, Campus de Beaulieu, 35042 Rennes, France
}

\begin{abstract}
The stability of the Kalman filter is usually ensured by the uniform complete controllability regarding the process noise and the uniform complete observability of linear time varying systems. This paper studies the case of continuous time output error systems, in which the process noise is totally absent. The classical stability analysis assuming the controllability regarding the process noise is thus not applicable. It is shown in this paper that the uniform complete observability alone is sufficient to ensure the asymptotic stability of the Kalman filter applied to time varying output error systems, regardless of the stability of the considered systems themselves. The exponential or polynomial convergence of the Kalman filter is then further analyzed for particular cases of stable or unstable output error systems.
\end{abstract}

Keywords: Kalman filter, Stability, Output error system.

\section{Introduction}

The well known Kalman filter has been extensively studied and is being applied in many different fields (Anderson and Moore, 1979; Jazwinski, 1970; Zarchan and Musof, 2005; Kim, 2011; Grewal and Andrews, 2015). The purpose of the present paper is to study the stability of the Kalman filter in a particular case rarely covered in the literature: the absence of process noise in the state equation of a linear time varying (LTV) system. Such systems are known as output error (OE) systems. Though typically process noise and output noise are both considered in Kalman filter applications, the case with no process noise is of particular interest when state equations originate from physical laws that are believed accurate enough. Another motivation of this study is OE system identification (Goodman and Dudley, 1987; Forssell and Ljung, 2000; Wang et al., 2015). When the prediction error method (Ljung, 1999) is applied to OE system identification, the stability of the output predictor is a crucial issue. The results presented in this paper ensure the stability of the predictor based on the Kalman filter.

While the optimal properties of the Kalman filter are frequently recalled, its stability properties are less often mentioned in the recent literature. The classical stability analysis is based on both the uniform complete controllability regarding the process noise and the uniform complete observability of LTV systems (Kalman, 1963; Jazwinski, 1970). In the case of OE systems, there is no process noise at all in the state equation, hence the controllability regarding the process

Email address: Qinghua.Zhang@inria.fr (Qinghua Zhang) 
noise cannot be fulfilled, and the classical stability results are not applicable. The present paper aims at completing this missing case.

The optimal state estimation realized by the Kalman filter can be viewed as a trade-off between the uncertainties in the state equation and in the output equation. In an OE system, the state equation is assumed noise-free. This point of view suggests that the state estimation should solely rely on the state equation, provided that the initial state of the OE system is exactly known. In practice the Kalman filter remains useful when the initial state is not exactly known or when the OE system is unstable. Of course, if the state of an unstable system diverges, so does its state estimate by the Kalman filter. Typically in practice, unstable systems are stabilized by feedback controllers so that the system state remains bounded. The Kalman filter can be applied either to the controlled system itself or to the entire closed loop system. In the latter case, the controller must be linear and completely known, excluding the saturation protection and any other nonlinearities.

The classical optimality results of the Kalman filter are also valid in the case of OE systems (Jazwinski, 1970, chapter 7). However, it is necessary to complete the stability analysis, as the classical results are not applicable in this case.

The main results presented in this paper are as follows. Under the uniform complete observability condition, it is first shown that the dynamics of the Kalman filter applied to a continuous time OE LTV system is asymptotically stable, regardless of the stability of the system itself. The exponential or polynomial convergence of the Kalman filter is further established, depending on the stability or the instability property of the considered system. The boundedness of the solution of the Riccati equation, and thus also of the Kalman gain, is also proved under the same condition. These results complete the classical results (Kalman, 1963; Jazwinski, 1970), which exclude the case of OE systems.

For linear time invariant (LTI) systems, it is a common practice to design the Kalman filter by solving an algebraic Riccati equation (in contrast to dynamic differential Riccati equation for general LTV systems as considered in the present paper). In this case, the controllability and observability conditions can be replaced by the weaker stabilizability and detectability conditions (Laub, 1979; Arnold and Laub, 1984).

Some preliminary results about the Riccati equation of time invariant systems have been presented in the conference paper (Ni and Zhang, 2013), and some further results on the asymptotic stability of the Kalman filter have been presented in the conference paper (Ni and Zhang, 2015), without the exponential or polynomial convergence rate analysis and part of the numerical examples reported in the present paper.

The rest of the paper is organized as follows. Some preliminary elements are introduced in Section 2. The problem considered in this paper is formulated in Section 3. The asymptotic stability of the Kalman filter for $\mathrm{OE}$ systems is established in Section 4. Exponential and polynomial convergence rates of the Kalman filter are analyzed in Section 5. Some analytical and numerical examples are presented in Sections 6 and 7. Finally, concluding remarks are drawn in Section 8.

\section{Definitions and preliminary elements}

Let us shortly recall some definitions and basic facts about LTV systems, which are necessary for the following sections.

Let $m$ and $n$ be any two positive integers. For a vector $x \in \mathbb{R}^{n},\|x\|$ denotes its Euclidean 
norm. For a matrix $A \in \mathbb{R}^{m \times n},\|A\|$ denotes the matrix norm induced by the Euclidean vector norm, which is equal to the largest singular value of $A$ and known as the spectral norm when $m=n$. Then $\|A x\| \leq\|A\|\|x\|$ for all $A \in \mathbb{R}^{m \times n}$ and all $x \in \mathbb{R}^{n}$. For two real square symmetric positive definite matrices $A$ and $B, A>B$ means $A-B$ is positive definite.

Let $A(t) \in \mathbb{R}^{m \times n}$ be defined for $t \in \mathbb{R}$. It is said (upper) bounded if $\|A(t)\|$ is bounded.

Consider the homogeneous LTV system

$$
\frac{d x(t)}{d t}=A(t) x(t)
$$

with $x(t) \in \mathbb{R}^{n}$ and $A(t) \in \mathbb{R}^{n \times n}$, and let $\Phi\left(t, t_{0}\right)$ be the associated state transition matrix such that, for all $t, t_{0} \in \mathbb{R}, d \Phi\left(t, t_{0}\right) / d t=A(t) \Phi\left(t, t_{0}\right)$ and $\Phi(t, t)=I_{n}$ with $I_{n}$ denoting the $n \times n$ identity matrix.

Definition 1. System (1) is Lyapunov stable if there exists a positive constant $\gamma$ such that, for all $t, t_{0} \in \mathbb{R}$ satisfying $t \geq t_{0}$, the following inequality holds

$$
\left\|\Phi\left(t, t_{0}\right)\right\| \leq \gamma
$$

This definition concerns the boundedness of the state vector, whereas the following definition ensures its convergence to zero.

Definition 2. System (1) is asymptotically stable if it is Lyapunov stable and if the following limiting behavior holds

$$
\lim _{t \rightarrow+\infty}\|x(t)\|=0
$$

for any initial state $x\left(t_{0}\right) \in \mathbb{R}^{n}$.

To characterize the convergence rate of some stable systems, the exponential stability is defined as follows.

Definition 3. System (1) is exponentially stable if there exist two positive constants $\alpha, \beta$ such that the inequality

$$
\left\|\Phi\left(t, t_{0}\right)\right\| \leq \beta e^{-\alpha\left(t-t_{0}\right)}
$$

holds for all $t, t_{0} \in \mathbb{R}$ satisfying $t \geq t_{0}$.

The definitions of Lyapunov and exponential stabilities in Definitions 1 and 3 are based on the norm of the state transition matrix $\Phi\left(t, t_{0}\right)$. They could take another equivalent form, as suggested by the following lemma. See also (Rugh, 1995).

Lemma 1. Let $M \in \mathbb{R}^{n \times n}$ and $\xi$ be some positive scalar value. Then $\|M\| \leq \xi$ if and only if $\|M v\| \leq \xi\|v\|$ for all $v \in \mathbb{R}^{n}$.

See the appendix at the end of this paper for a proof of the lemma.

With $M=\Phi\left(t, t_{0}\right), \xi=\gamma$ or $\xi=\beta e^{-\alpha\left(t-t_{0}\right)}$, Lemma 1 suggests that Definitions 1 and 3 could be based on the norm of $x(t)=\Phi\left(t, t_{0}\right) x\left(t_{0}\right)$ instead of the norm of $\Phi\left(t, t_{0}\right)$. 
Definition 4. System (1) is strongly unstable if there exist two positive constants $\alpha, \beta$ such that the inequality

$$
\|x(t)\| \geq \beta e^{\alpha\left(t-t_{0}\right)}\left\|x\left(t_{0}\right)\right\|
$$

holds for all $t, t_{0} \in \mathbb{R}$ satisfying $t \geq t_{0}$ and for all $x\left(t_{0}\right) \in \mathbb{R}^{n}$.

The following observability definition for LTV systems follows (Kalman, 1963).

Definition 5. The matrix pair $[A(t), C(t)]$ with $A(t) \in \mathbb{R}^{n \times n}$ and $C(t) \in \mathbb{R}^{m \times n}$ is uniformly completely observable if there exist positive constants $\tau, \rho_{1}$ and $\rho_{2}$ such that, for all $t \in \mathbb{R}$, the following inequalities hold ${ }^{1}$

$$
\begin{aligned}
\rho_{1} I_{n} & \leq \int_{t-\tau}^{t} \Phi^{T}(s, t) C^{T}(s) R^{-1}(s) C(s) \Phi(s, t) d s \\
& \leq \rho_{2} I_{n}
\end{aligned}
$$

with some bounded symmetric positive definite matrix $R(s) \in \mathbb{R}^{m \times m}$ (typically the covariance matrix of the output noise in a stochastic state space system).

The fact that observability is preserved by output feedback is stated in the following lemma.

Lemma 2. Let $A(t) \in \mathbb{R}^{n \times n}, C(t) \in \mathbb{R}^{m \times n}, K(t) \in \mathbb{R}^{n \times m}$ be bounded piecewise continuous matrices. The matrix pair $[A(t), C(t)]$ is uniformly completely observable, if and only if the matrix pair $[A(t)-K(t) C(t), C(t)]$ is uniformly completely observable.

This lemma can be found in (Anderson et al., 1986, chapter 2). For more rigorous proofs see (Sastry and Bodson, 1989, chapter 2), (Ioannou and Sun, 1996, chapter 4), and (Zhang and Zhang, 2015).

\section{Problem formulation and assumptions}

In this section the Kalman filter and its usual assumptions are first recalled, before the presentation of the particular case of OE systems considered in this paper.

\subsection{Kalman filter and usual assumptions}

The Kalman filter in continuous-time is usually designed for LTV systems modeled by

$$
\begin{aligned}
& d x(t)=A(t) x(t) d t+B(t) u(t) d t+Q^{\frac{1}{2}}(t) d \omega(t) \\
& d y(t)=C(t) x(t) d t+R^{\frac{1}{2}}(t) d \eta(t)
\end{aligned}
$$

where $t \in \mathbb{R}$ represents the time, $x(t) \in \mathbb{R}^{n}$ is the state vector, $u(t) \in \mathbb{R}^{l}$ the bounded input, $y(t) \in \mathbb{R}^{m}$ the output, $\omega(t) \in \mathbb{R}^{n}, \eta(t) \in \mathbb{R}^{m}$ are two independent Brownian processes with identity covariance matrices, $A(t), B(t), C(t), Q(t), R(t)$ are bounded real matrices of appropriate sizes. The matrix $Q(t)$ is bounded and symmetric positive semi-definite, whereas $R(t)$ is bounded and symmetric positive definite. The notations $Q^{\frac{1}{2}}(t)$ and $R^{\frac{1}{2}}(t)$ denote respectively

\footnotetext{
${ }^{1}$ Some variants of the definition of the uniform complete observability exist in the literature. The definition recalled here follows (Kalman, 1963).
} 
the symmetric positive (semi)-definite matrix square roots of $Q(t)$ and $R(t)$. The initial state $x\left(t_{0}\right) \in \mathbb{R}^{n}$ is a random vector following the Gaussian distribution $x\left(t_{0}\right) \sim \mathcal{N}\left(x_{0}, P_{0}\right)$ with $x_{0} \in \mathbb{R}^{n}$ and $P_{0} \in \mathbb{R}^{n \times n}$.

The Kalman filter for this LTV system is as follows:

$$
\begin{aligned}
& d \hat{x}(t)=A(t) \hat{x}(t) d t+B(t) u(t) d t+K(t)(d y(t)-C(t) \hat{x}(t) d t) \\
& K(t)=P(t) C^{T}(t) R^{-1}(t) \\
& \frac{d}{d t} P(t)=A(t) P(t)+P(t) A^{T}(t)-P(t) C(t)^{T} R^{-1}(t) C(t) P(t)+Q(t) \\
& \hat{x}\left(t_{0}\right)=x_{0}, \quad P\left(t_{0}\right)=P_{0}
\end{aligned}
$$

where the solution of the Riccati equation (9c) is a matrix function $P(t) \in \mathbb{R}^{n \times n}$, and $K(t) \in$ $\mathbb{R}^{n \times m}$ is the Kalman gain .

The optimal properties of the Kalman filter are well known. It also known that the solution $P(t)$ of the Riccati equation is bounded and that the dynamics of the Kalman filter is stable, provided that the matrix pair $\left[A(t), Q^{\frac{1}{2}}(t)\right]$ is uniformly completely controllable and the matrix pair $[A(t), C(t)]$ is uniformly completely observable (Kalman, 1963; Jazwinski, 1970). These are obviously crucial properties in practice for online algorithms.

It is worth mentioning that, in (Kalman, 1963; Jazwinski, 1970), the proofs of these stability results are based on an important lemma, which turns out to be incorrect, as pointed out independently by the authors of (Delyon, 2001; Pengov et al., 2001). Fortunately, the mistake has been repaired in these more recent references so that the main classical results remain correct.

\subsection{Output error systems and Kalman filter}

In the case of output error (OE) systems, the process noise is absent from the state equation, hence the general LTV system (8) becomes

$$
\begin{aligned}
& d x(t)=A(t) x(t) d t+B(t) u(t) d t \\
& d y(t)=C(t) x(t) d t+R^{\frac{1}{2}}(t) d \eta(t) .
\end{aligned}
$$

An OE system can be seen as a particular LTV system (8) with $Q(t) \equiv 0$. The Kalman filter (9) then becomes

$$
\begin{aligned}
& d \hat{x}(t)=A(t) \hat{x}(t) d t+B(t) u(t) d t+K(t)(d y(t)-C(t) \hat{x}(t) d t) \\
& K(t)=P(t) C^{T}(t) R^{-1}(t) \\
& \frac{d}{d t} P(t)=A(t) P(t)+P(t) A^{T}(t)-P(t) C(t)^{T} R^{-1}(t) C(t) P(t) \\
& \hat{x}\left(t_{0}\right)=x_{0}, \quad P\left(t_{0}\right)=P_{0} .
\end{aligned}
$$

In this case, the classical optimality results of the Kalman filter remain valid (Jazwinski, 1970, chapter 7). Nevertheless, the uniform complete controllability condition cannot be satisfied by

the matrix pair $\left[A(t), Q^{\frac{1}{2}}(t)\right]$, as $Q^{\frac{1}{2}}(t) \equiv 0$. Consequently, the classical results on the stability of the Kalman filter cannot be applied here. The main purpose of the present paper is to study the Kalman filter stability in this particular case.

\subsection{General assumptions}

The assumptions stated here are required for all the following sections of this paper. 
The considered OE system (10) is defined with bounded and piecewise continuous real matrices $A(t), B(t), C(t), R(t)$ of appropriate sizes, and in particular, with a symmetric positive definite $R(t)$. It is also assumed that $R^{-1}(t)$ is bounded.

The initial state $x\left(t_{0}\right) \in \mathbb{R}^{n}$ is a random vector following the Gaussian distribution

$$
x\left(t_{0}\right) \sim \mathcal{N}\left(x_{0}, P_{0}\right) .
$$

with some known mean vector $x_{0} \in \mathbb{R}^{n}$ and symmetric positive definite covariance matrix $P_{0} \in \mathbb{R}^{n \times n}$.

It is further assumed that the matrix pair $[A(t), C(t)]$ is uniformly completely observable (see Definition 5).

\section{Asymptotic stability of the Kalman filter for output error systems}

In this section will be established the asymptotic stability of the Kalman filter (11) for all OE system (10), regardless of the stability of the OE system itself. The convergence type in particular cases will be further analyzed in the next section.

\subsection{Behavior of the solution of the Riccati equation}

Let us first establish the positive definiteness and the upper boundedness of $P(t)$, which are of crucial importance in practice, before studying the asymptotic stability of the Kalman filter.

It will be shown that the solution of the Riccati equation (11c) is closely related to the solution of the following Lyapunov equation

$$
\begin{aligned}
& \frac{d \Omega(t)}{d t}+A^{T}(t) \Omega(t)+\Omega(t) A(t)=C^{T}(t) R^{-1}(t) C(t) \\
& \Omega\left(t_{0}\right)=P_{0}^{-1}
\end{aligned}
$$

with the same matrices $A(t), C(t), R(t), P_{0}$ as in (11c) and (11d).

Proposition 1. The solution $\Omega(t) \in \mathbb{R}^{n \times n}$ of the Lyapunov equation (13) is a symmetric positive definite matrix for all $t \geq t_{0}$.

Proof. It can be directly checked that

$$
\Omega(t)=\Phi^{T}\left(t_{0}, t\right) \Omega\left(t_{0}\right) \Phi\left(t_{0}, t\right)+\int_{t_{0}}^{t} \Phi^{T}(s, t) C^{T}(s) R^{-1}(s) C(s) \Phi(s, t) d s
$$

satisfies the Lyapunov equation (13). As $\Omega\left(t_{0}\right)=P_{0}^{-1}$ is positive definite, $\Phi\left(t_{0}, t\right)$ is an invertible matrix, and for all $t \geq t_{0}$ the integral in (14) is positive semidefinite, therefore, $\Omega(t)$ is positive definite for all $t \geq t_{0}$.

Now it is certain that, for all $t \geq t_{0}, \Omega(t)$ is positive definite, thus invertible. By applying the matrix differentiation rule

$$
\frac{d \Omega^{-1}(t)}{d t}=-\Omega^{-1}(t) \frac{d \Omega(t)}{d t} \Omega^{-1}(t)
$$

the following result can be directly checked.

Proposition 2. Let $\Omega(t)$ be the solution the Lyapunov equation (13) for $t \geq t_{0}$, then $P(t) \triangleq$ $\Omega^{-1}(t)$ solves the Riccati equation (11c) with the initial condition $P\left(t_{0}\right)=P_{0}$. 
It is then clear that the properties of $P(t)$ can be studied through those of $\Omega(t)$.

Proposition 3. Under the assumptions stated in Section 3.3, for all $t \geq t_{0}$, the solution $P(t)$ of the Riccati equation (11c) with the initial condition $P\left(t_{0}\right)=P_{0}>0$ is symmetric positive definite and is upper bounded.

Proof. The positive definiteness of $P(t)$ is immediate from that of $\Omega(t)=P^{-1}(t)$. To show that $P(t)$ is upper bounded, it will be shown that $\Omega(t)$ is lower bounded.

First consider the case $t \in\left[t_{0}, t_{0}+\tau\right]$ (remind that $\tau$ is the positive constant involved in the observability condition (6)). In this finite time interval, the first term in (14) is positive definite, and the second term is positive semidefinite, then

$$
\Omega(t) \geq \rho_{0} I_{n}
$$

where $\rho_{0}>0$ is the minimum of the smallest singular value of the matrix $\Phi^{T}\left(t_{0}, t\right) \Omega\left(t_{0}\right) \Phi\left(t_{0}, t\right)$ for $t \in\left[t_{0}, t_{0}+\tau\right]$.

It remains to consider the case $t>t_{0}+\tau$. Let

$$
N \triangleq\left[\frac{t-t_{0}}{\tau}\right]
$$

be the largest integer smaller than or equal to $\left(t-t_{0}\right) / \tau$. For the second term of (14), decompose the integral into the sum of $N$ integrals over intervals of size $\tau$, possibly dropping the part of the integral at the beginning of $\left[t_{0}, t_{0}+\tau\right]$ smaller than $\tau$ if $\left(t-t_{0}\right) / \tau$ is not exactly an integer. It then yields

$$
\begin{aligned}
& \int_{t_{0}}^{t} \Phi^{T}(s, t) C^{T}(s) R^{-1}(s) C(s) \Phi(s, t) d s \\
& \geq \sum_{k=0}^{N-1} \int_{t-(k+1) \tau}^{t-k \tau} \Phi^{T}(s, t) C^{T}(s) R^{-1}(s) C(s) \Phi(s, t) d s \\
& =\sum_{k=0}^{N-1} \Phi^{T}(t-k \tau, t) S_{k} \Phi(t-k \tau, t)
\end{aligned}
$$

with

$$
S_{k} \triangleq \int_{t-(k+1) \tau}^{t-k \tau} \Phi^{T}(s, t-k \tau) C^{T}(s) R^{-1}(s) C(s) \Phi(s, t-k \tau) d s .
$$

Each $S_{k}$ (for $k=0,1, \ldots, N-1$ ) corresponds to the integral in the uniform complete observability condition (6), which holds for all $t \in \mathbb{R}$. Hence $S_{k} \geq \rho_{1} I_{n}$ for $k=0,1, \ldots, N-1$, with the positive constant $\rho_{1}$ as in (6). Then

$$
\int_{t_{0}}^{t} \Phi^{T}(s, t) C^{T}(s) R^{-1}(s) C(s) \Phi(s, t) d s \geq \rho_{1} \sum_{k=0}^{N-1} \Phi^{T}(t-k \tau, t) \Phi(t-k \tau, t) .
$$

Now in the sum of (19) keep only the term with $k=0$ (the other terms will be useful for proving other results). Then 


$$
\begin{aligned}
& \int_{t_{0}}^{t} \Phi^{T}(s, t) C^{T}(s) R^{-1}(s) C(s) \Phi(s, t) d s \\
& \geq \rho_{1} \Phi^{T}(t, t) \Phi(t, t) \\
& \geq \rho_{1} I_{n} .
\end{aligned}
$$

It means that the second term of (14) is lower bounded by $\rho_{1} I_{n}$ for $t>t_{0}+\tau$. By summarizing the results for the cases $t \in\left[t_{0}, t_{0}+\tau\right]$ and $t>t_{0}+\tau$, it is thus shown that $\Omega(t)$ is lower bounded by a strictly positive definite matrix for all $t \geq t_{0}$. To be more specific,

$$
\Omega(t) \geq \min \left(\rho_{0}, \rho_{1}\right) I_{n}, \forall t \geq t_{0} .
$$

It is then concluded that $P(t)=\Omega^{-1}(t)$ is upper bounded for all $t \geq t_{0}$.

\subsection{The asymptotic stability of the Kalman filter}

For the OE system Kalman filter (11), it is already shown that the matrix $P(t)$ is upper bounded, it then follows from the assumptions about the boundedness of $C(t)$ and $R^{-1}(t)$ that the Kalman gain $K(t)$ is also upper bounded. Equation (11a) governing $\hat{x}(t)$ can be viewed as a dynamic system driven by the "exogenous input" terms $B(t) u(t) d t$ and $K(t) d y$. The intrinsic stability property of (11a) is related to its homogeneous part, namely the LTV system

$$
\frac{d}{d t} z(t)=(A(t)-K(t) C(t)) z(t)
$$

with $z(t) \in \mathbb{R}^{n}$. Like in (Jazwinski, 1970), when the stability of the Kalman filter is talked about, it refers to the stability of this homogeneous LTV system.

Now it is ready to present the main result of this section.

Theorem 1. Under the assumptions stated in Section 3.3, the homogeneous part of the OE system Kalman filter state estimation equation (11a), as expressed in equation (24), is asymptotically stable.

As mentioned above, the stability of the homogeneous part of the Kalman filter is an intrinsic stability property independent of the signals being processed by the filter. It implies that the mathematical expectation of the state estimation error is asymptotically stable. Moreover, the boundedness of the covariance matrix of the state estimation error, namely $P(t)$, is ensued by Proposition 3.

\section{Proof of Theorem 1}

Define the Lyapunov function candidate

$$
V(z(t), t) \triangleq z^{T}(t) \Omega(t) z(t)
$$

with the positive definite $\Omega(t)$ as defined in (13).

Compute the derivative of $V(z(t), t)$ along the trajectory of $(24)$,

$$
\begin{aligned}
\frac{d V(z(t), t)}{d t} & =z^{T}(t)\left((A(t)-K(t) C(t))^{T} \Omega(t)\right. \\
& \left.+\Omega(t)(A(t)-K(t) C(t))+\frac{d \Omega(t)}{d t}\right) z(t) .
\end{aligned}
$$


Remind that $\Omega(t)$ satisfies (13a), then

$$
\begin{aligned}
\frac{d V(z(t), t)}{d t} & =z^{T}(t)\left((-K(t) C(t))^{T} \Omega(t)\right. \\
& \left.+\Omega(t)(-K(t) C(t))+C^{T}(t) R^{-1}(t) C(t)\right) z(t) .
\end{aligned}
$$

In (11b) replace $P(t)$ by $\Omega^{-1}(t)$, then

$$
K(t)=\Omega^{-1}(t) C^{T}(t) R^{-1}(t),
$$

hence

$$
\frac{d V(z(t), t)}{d t}=-z^{T}(t) C^{T}(t) R^{-1}(t) C(t) z(t)
$$

which is negative semidefinite ${ }^{2}$. It is then clear that the value of $V(z(t), t)$ cannot increase with the time $t \geq t_{0}$, thus

$$
V(z(t), t) \leq V\left(z\left(t_{0}\right), t_{0}\right), \quad \forall t \geq t_{0} .
$$

It then follows from the definition of $V(z(t), t)$ that

$$
z^{T}(t) \Omega(t) z(t) \leq z^{T}\left(t_{0}\right) \Omega\left(t_{0}\right) z\left(t_{0}\right) .
$$

Let $\sigma_{0}$ be the smallest singular value of $P_{0}$, then $\sigma_{0}^{-1}$ is the largest singular value of $\Omega\left(t_{0}\right)=P_{0}^{-1}$. Remind the lower bound of $\Omega(t)$ for all $t \geq t_{0}$ as shown in (23), then

$$
\begin{aligned}
\min \left(\rho_{0}, \rho_{1}\right)\|z(t)\|^{2} & \leq z^{T}(t) \Omega(t) z(t) \\
& \leq z^{T}\left(t_{0}\right) \Omega\left(t_{0}\right) z\left(t_{0}\right) \leq \sigma_{0}^{-1}\left\|z\left(t_{0}\right)\right\|^{2} .
\end{aligned}
$$

Hence

$$
\|z(t)\| \leq \frac{1}{\sqrt{\sigma_{0} \min \left(\rho_{0}, \rho_{1}\right)}}\left\|z\left(t_{0}\right)\right\|
$$

holds for all $z\left(t_{0}\right) \in \mathbb{R}^{n}$. Therefore the homogeneous system (24) is Lyapunov stable (see Definition 1 and Lemma 1).

To show the asymptotic stability of (24), the limiting behavior of $z(t)$ when $t \rightarrow+\infty$ will be analyzed.

Let the state transition matrix of the homogeneous LTV system (24) be denoted by $\Phi_{K}(s, t)$, then $z(s)=\Phi_{K}(s, t) z(t)$ for any $s, t \in \mathbb{R}$. It then follows from $(27)$ that

$$
\frac{d V(z(s), s)}{d s}=-z^{T}(t) \Phi_{K}^{T}(s, t) C^{T}(s) R^{-1}(s) C(s) \Phi_{K}(s, t) z(t)
$$

and therefore

\footnotetext{
${ }^{2}$ Note that in the classical case (Kalman, 1963; Jazwinski, 1970) where a uniformly completely controllable and observable system is considered, the solution $P(t)$ of the Riccati equation is shown both upper and lower bounded by strictly positive definite matrices, and moreover, there is one more term, $-z^{T}(t) P^{-1}(t) Q(t) P^{-1}(t) z(t)$, at the right hand side of (27), which helps to establish the convergence to zero of $V(z(t), t)$. The matrix $C^{T}(t) R^{-1}(t) C(t)$ in (27) is typically rank deficient at each instant $t$ (typically $C(t)$ has less rows than columns).
} 


$$
\begin{aligned}
& V(z(t), t)-V(z(t-\tau), t-\tau) \\
& =\int_{t-\tau}^{t} \frac{d V(z(s), s)}{d s} d s \\
& =-z^{T}(t) O_{K}(t, t-\tau) z(t)
\end{aligned}
$$

with

$$
O_{K}(t, t-\tau) \triangleq \int_{t-\tau}^{t} \Phi_{K}^{T}(s, t) C^{T}(s) R^{-1}(s) C(s) \Phi_{K}(s, t) d s
$$

which is the observability Gramian matrix of the matrix pair $[A(t)-K(t) C(t), C(t)]$. The fact that $P(t), C(t), R^{-1}(t)$ are all upper bounded implies that the Kalman gain $K(t)$ is also bounded (see Proposition 3 and its proof). According to Lemma 2 (see Section 2), the assumed uniform complete observability of the matrix pair $[A(t), C(t)]$ (see Section 3.3) implies that the matrix pair $[A(t)-K(t) C(t), C(t)]$ is also uniformly completely observable, hence there exists a positive constant $\rho_{3}$ such that

$$
0<\rho_{3} I_{n} \leq O_{K}(t, t-\tau) .
$$

This result, together with $(32)$, leads to

$$
V(z(t), t) \leq V(z(t-\tau), t-\tau)-\rho_{3}\|z(t)\|^{2} .
$$

It means that, over each time interval of size $\tau$, the value of $V(z(t), t)$ is decreased by $\rho_{3}\|z(t)\|^{2} \geq$ 0 .

Though $V(z(t), t)$ is decreased over each time interval of size $\tau$, it may not necessarily tend to zero, as the decrement may be infinitesimal.

It will be shown that $\|z(t)\|$ tends to zero by means of contradiction.

For the purpose of proof by contradiction, assume that $\|z(t)\|$ does not tend to zero when $t \rightarrow+\infty$. Then there exists a constant $\varepsilon>0$, such that for any (arbitrarily large) $T \in \mathbb{R}$, there exists $t>T$ such that $\|z(t)\|>\varepsilon$, therefore

$$
V(z(t), t) \leq V(z(t-\tau), t-\tau)-\rho_{3} \varepsilon^{2} .
$$

There exist infinitely many such values of $t$, as $T$ can be arbitrarily large. Moreover, it was already shown that the value of $V(z(t), t)$ cannot increase with the time $t$. Inequality (35) then says that $V(z(t), t)$ is repeatedly decreased by $\rho_{3} \varepsilon^{2}>0$ for larger and larger values of $t$. Consequently, $V(z(t), t)$ will become negative for sufficiently large values of $t$. This is in contradiction with the definition of $V(z(t), t)$ in $(25)$ which is positive definite. Therefore, it is proved that $\|z(t)\|$ tends to zero when $t \rightarrow+\infty$.

The asymptotic stability of the homogeneous system (24) is then established.

\section{Convergence rates of the Kalman filter}

In the previous section, the asymptotic convergence of the Kalman filter was established in the general case of $\mathrm{OE}$ systems, regardless of the stability of the considered system itself. The main condition was the uniform complete observability, in addition to the usual regularity assumptions on the system matrices.

In this section, it will be further shown that the homogeneous part of the Kalman filter, namely system (24), converges with exponential or polynomial rates in some cases, depending on the stability or the instability of the considered OE system (10). 
Three cases will be considered, each corresponding to exponentially stable, Lyapunov stable or strongly unstable OE systems.

\subsection{Exponentially stable output error systems}

In this subsection it is assumed that the considered OE system (10) is exponentially stable. The following quite natural result will be established.

Theorem 2. Under the assumptions stated in Section 3.3, if the considered OE system (10) is exponentially stable, then the homogeneous part of the Kalman filter, namely system (24), is also exponentially stable.

Proof. Now the OE system (10) is assumed exponentially stable (see Definition 3), there exit positive constants $\alpha, \beta$ such that $\|\Phi(t, s)\| \leq \beta e^{-\alpha(t-s)}$ for all $t, s \in \mathbb{R}$ satisfying $t \geq s$, then

$$
\left\|\Phi^{-1}(s, t)\right\|=\|\Phi(t, s)\| \leq \beta e^{-\alpha(t-s)} .
$$

Let $\sigma_{1}(s, t)>0$ denote the smallest singular value of $\Phi(s, t)$, then $1 / \sigma_{1}(s, t)$ is the largest singular value of $\Phi^{-1}(s, t)$, thus

$$
0<\frac{1}{\sigma_{1}(s, t)}=\left\|\Phi^{-1}(s, t)\right\| \leq \beta e^{-\alpha(t-s)}
$$

or equivalently

$$
\sigma_{1}(s, t) \geq \frac{1}{\beta} e^{\alpha(t-s)} .
$$

As $\sigma_{1}(s, t)$ is the smallest singular value of $\Phi(s, t)$,

$$
\Phi^{T}(s, t) \Phi(s, t) \geq \sigma_{1}^{2}(s, t) I_{n} \geq \frac{1}{\beta^{2}} e^{2 \alpha(t-s)} I_{n}
$$

Let us go back to (19), in which each term of the sum is positive definite. Keep only the term corresponding to $k=N-1$ (remind that $\left.N=\left[\left(t-t_{0}\right) / \tau\right]\right)$, then

$$
\begin{aligned}
\int_{t_{0}}^{t} \Phi^{T}(s, t) & C^{T}(s) R^{-1}(s) C(s) \Phi(s, t) d s \\
& \geq \rho_{1} \Phi^{T}(t-(N-1) \tau, t) \Phi(t-(N-1) \tau, t) \\
& \geq \rho_{1} \frac{1}{\beta^{2}} e^{2 \alpha(N-1) \tau} I_{n}
\end{aligned}
$$

where the last inequality results from the application of (38) with $s=t-(N-1) \tau$.

In (14) the first term of the right hand side is positive definite, then

$$
\begin{aligned}
\Omega(t) & \geq \int_{t_{0}}^{t} \Phi^{T}(s, t) C^{T}(s) R^{-1}(s) C(s) \Phi(s, t) d s \\
& \geq \frac{\rho_{1}}{\beta^{2}} e^{2 \alpha(N-1) \tau} I_{n} .
\end{aligned}
$$

This result implies that the function $V(z(t), t)$ defined in $(25)$ satisfies

$$
V(z(t), t) \geq \frac{\rho_{1}}{\beta^{2}} e^{2 \alpha(N-1) \tau}\|z(t)\|^{2} .
$$

For all $t \geq t_{0}$, the already established inequality (28) leads to 


$$
V\left(z\left(t_{0}\right), t_{0}\right) \geq V(z(t), t) \geq \frac{\rho_{1}}{\beta^{2}} e^{2 \alpha(N-1) \tau}\|z(t)\|^{2}
$$

therefore

$$
\|z(t)\|^{2} \leq \frac{\beta^{2}}{\rho_{1}} e^{-2 \alpha(N-1) \tau} V\left(z\left(t_{0}\right), t_{0}\right) .
$$

Remind that $N=\left[\left(t-t_{0}\right) / \tau\right]$, therefore

$$
N>\frac{t-t_{0}}{\tau}-1
$$

and then

$$
\begin{aligned}
\|z(t)\|^{2} & <\frac{\beta^{2}}{\rho_{1}} e^{-2 \alpha\left(t-t_{0}-2 \tau\right)} V\left(z\left(t_{0}\right), t_{0}\right) \\
& =\frac{\beta^{2}}{\rho_{1}} e^{-2 \alpha\left(t-t_{0}-2 \tau\right)} z^{T}\left(t_{0}\right) \Omega\left(t_{0}\right) z\left(t_{0}\right) \\
& \leq \frac{\beta^{2}}{\rho_{1}} e^{-2 \alpha\left(t-t_{0}-2 \tau\right)} \rho_{4}\left\|z\left(t_{0}\right)\right\|^{2}
\end{aligned}
$$

where $\rho_{4}>0$ denotes the largest singular value of $\Omega\left(t_{0}\right)$.

Note that this result holds for any $z\left(t_{0}\right) \in \mathbb{R}^{n}$ and the corresponding $z(t)$, the exponential convergence of the homogeneous part of the Kalman filter is thus proved.

\subsection{Lyapunov stable output error systems}

Now let us consider the OE systems (10) which are Lyapunov stable (see Definition 1). This class of systems is wider than and includes the exponentially stable systems considered in the previous subsection.

Theorem 3. Under the assumptions stated in Section 3.3, if the considered OE system (10) is Lyapunov stable, then there exists a positive constant $\mu$ such that the state $z(t)$ of the homogeneous part of the Kalman filter, namely system (24), satisfies $\|z(t)\|^{2} \leq\left(1 /\left(\mu\left(t-t_{1}\right)\right)\right)\left\|z\left(t_{1}\right)\right\|^{2}$ for any $z\left(t_{1}\right) \in \mathbb{R}^{n}$ at $t_{1} \triangleq t_{0}+\tau$ and any $t \geq t_{1}$.

This result states that $\|z(t)\|^{2}$ converges to zero with a speed not slower than $1 /\left(\mu\left(t-t_{1}\right)\right)$.

Proof. The proof of Theorem 3 is quite similar to that of Theorem 2. From the beginning of the proof to the inequality (38), the only modification is to replace $\beta e^{-\alpha(t-s)}$ by $\gamma$ for the upper bound of $\|\Phi(t, s)\|$, with $\gamma$ being the upper bound introduced in (2). Then (38) becomes

$$
\Phi^{T}(s, t) \Phi(s, t) \geq \frac{1}{\gamma^{2}} I_{n}
$$

Now let us go back to (19). As $k \tau \geq 0$, each term in the sum of (19) is lower bounded by $\left(1 / \gamma^{2}\right) I_{n}$, by applying (48). Then (19) and (48) lead to 


$$
\begin{aligned}
\int_{t_{0}}^{t} \Phi^{T}(s, t) & C^{T}(s) R^{-1}(s) C(s) \Phi(s, t) d s \\
& \geq \rho_{1} \sum_{k=0}^{N-1} \frac{1}{\gamma^{2}} I_{n} \\
& =\frac{N \rho_{1}}{\gamma^{2}} I_{n} .
\end{aligned}
$$

This result is the counterpart of (40) where $\Omega(t)$ increases exponentially in $N$, but in the present case it increases linearly in $N$. Then the following steps are similar to those in the proof of Theorem 2 after (40), with minor modifications. Notably, for $t \geq t_{1}$ with

$$
t_{1} \triangleq t_{0}+\tau
$$

the inequalities in (42) become

$$
V\left(z\left(t_{1}\right), t_{1}\right) \geq V(z(t), t) \geq \frac{N \rho_{1}}{\gamma^{2}}\|z(t)\|^{2}
$$

and the last step (47) becomes

$$
\|z(t)\|^{2} \leq \frac{\gamma^{2} \rho_{4} \tau}{\rho_{1}\left(t-t_{1}\right)}\left\|z\left(t_{1}\right)\right\|^{2} .
$$

Theorem 3 is then established with

$$
\mu=\frac{\rho_{1}}{\gamma^{2} \rho_{4} \tau}
$$

\subsection{Strongly unstable output error systems}

This subsection studies OE systems that are strongly unstable (see Definition 4). In the classical case where uniform controllability and observability are assumed (thus excluding OE systems), the stability of (the homogeneous part of) the Kalman filter was established, regardless of the stability of the considered system itself (Kalman, 1963; Jazwinski, 1970).

In the present paper, the asymptotic stability of the Kalman filter for OE systems proved in Section 4 is valid for all OE systems, including unstable systems. Here the exponential stability of the Kalman filter in this case will be further proved.

Theorem 4. Under the assumptions stated in Section 3.3, if the considered OE system (10) is strongly unstable, then the homogeneous part of the Kalman filter, namely system (24), is exponentially stable.

Proof. In the general result of Section 4, it was already shown in (23) that the the solution $\Omega(t)$ of the Lyapunov equation is lower bounded. Now let us show that in the present case of strongly unstable systems, $\Omega(t)$ is also upper bounded.

The definition of strong instability (see Definition 4) means

$$
\|\Phi(t, s) v\| \geq \beta e^{\alpha(t-s)}\|v\|
$$


for all $t, s \in \mathbb{R}$ satisfying $t \geq s$ and for all $v \in \mathbb{R}^{n}$. It implies that the smallest singular value of $\Phi(t, s)$, namely $\sigma_{1}(t, s)$, satisfies

$$
\sigma_{1}(t, s) \geq \beta e^{\alpha(t-s)} .
$$

The largest singular value of $\Phi^{-1}(t, s)=\Phi(s, t)$ is equal to $1 / \sigma_{1}(t, s)$ and satisfies

$$
\frac{1}{\sigma_{1}(t, s)} \leq \frac{1}{\beta} e^{-\alpha(t-s)}
$$

This result then implies

$$
\Phi^{T}(s, t) \Phi(s, t) \leq \frac{1}{\beta^{2}} e^{-2 \alpha(t-s)} I_{n}
$$

for all $t, s \in \mathbb{R}$ satisfying $t \geq s$.

Now let us go back to (14) to study the upper bound of $\Omega(t)$. Apply (54) to the first term at the right hand side of (14), with $\rho_{4}>0$ denoting the largest singular value of $\Omega\left(t_{0}\right)$, then for all $t, t_{0} \in \mathbb{R}$ satisfying $t \geq t_{0}$,

$$
\begin{aligned}
\Phi^{T}\left(t_{0}, t\right) \Omega\left(t_{0}\right) \Phi\left(t_{0}, t\right) & \leq \frac{\rho_{4}}{\beta^{2}} e^{-2 \alpha\left(t-t_{0}\right)} I_{n} \\
& \leq \frac{\rho_{4}}{\beta^{2}} I_{n} .
\end{aligned}
$$

For the integral term in (14), remind that $C(t)$ and $R^{-1}(t)$ are both bounded, then there exists a constant $\rho_{5}>0$ such that

$$
C^{T}(s) R^{-1}(s) C(s) \leq \rho_{5} I_{n}
$$

and

$$
\Phi^{T}(s, t) C^{T}(s) R^{-1}(s) C(s) \Phi(s, t) \leq \rho_{5} \Phi^{T}(s, t) \Phi(s, t) .
$$

Apply again (54), then the integral term in (14) satisfies, for $t \geq t_{0}$,

$$
\begin{aligned}
\int_{t_{0}}^{t} \Phi^{T}(s, t) C^{T}(s) R^{-1}(s) C(s) \Phi(s, t) d s \\
\quad \leq \rho_{5} I_{n} \int_{t_{0}}^{t} \frac{1}{\beta^{2}} e^{-2 \alpha(t-s)} d s \\
\quad=\frac{\rho_{5}}{2 \alpha \beta^{2}}\left(1-e^{-2 \alpha\left(t-t_{0}\right)}\right) I_{n} \\
\quad \leq \frac{\rho_{5}}{2 \alpha \beta^{2}} I_{n} .
\end{aligned}
$$

It then follows from (56) and (60) that

$$
\Omega(t) \leq\left(\frac{\rho_{4}}{\beta^{2}}+\frac{\rho_{5}}{2 \alpha \beta^{2}}\right) I_{n}
$$

This result implies that the function $V(z(t), t)$ defined in (25) satisfies

$$
V(z(t), t) \leq \frac{2 \alpha \rho_{4}+\rho_{5}}{2 \alpha \beta^{2}}\|z(t)\|^{2}
$$

It is then combined with (34) to obtain

$$
V(z(t), t) \leq V(z(t-\tau), t-\tau)-\frac{2 \rho_{3} \alpha \beta^{2}}{2 \alpha \rho_{4}+\rho_{5}} V(z(t), t)
$$


and therefore

$$
V(z(t), t) \leq q V(z(t-\tau), t-\tau)
$$

with

$$
0<q \triangleq \frac{1}{1+\frac{2 \rho_{3} \alpha \beta^{2}}{2 \alpha \rho_{4}+\rho_{5}}}<1 .
$$

This result means that over each time interval of size $\tau$, the value of $V(z(t), t)$ is multiplied by a factor not larger than $q<1$. Let $N$ be the integer as defined in (16), then

$$
\begin{aligned}
V(z(t), t) & \leq V\left(z\left(t_{0}\right), t_{0}\right) q^{N} \\
& <V\left(z\left(t_{0}\right), t_{0}\right) q^{\frac{t-t_{0}}{\tau}-1} .
\end{aligned}
$$

The lower bound of $\Omega(t)$ shown in (23) then implies

$$
\begin{aligned}
\|z(t)\|^{2} & \leq \frac{1}{\min \left(\rho_{0}, \rho_{1}\right)} V(z(t), t) \\
& <\frac{V\left(z\left(t_{0}\right), t_{0}\right)}{\min \left(\rho_{0}, \rho_{1}\right)} q^{\frac{t-t_{0}}{\tau}-1} \\
& \leq \frac{\rho_{4}}{\min \left(\rho_{0}, \rho_{1}\right)} q^{\frac{t-t_{0}}{\tau}-1}\left\|z\left(t_{0}\right)\right\|^{2}
\end{aligned}
$$

where $\rho_{4}>0$ is the largest singular value of $\Omega\left(t_{0}\right)$.

Remind that $0<q<1$, therefore $\|z(t)\|$ converges to zero exponentially.

\subsection{Other cases}

In this section the exponential or polynomial convergence of the Kalman filter has been proved for the cases of exponentially stable, Lyapunov stable or strongly unstable OE systems. These cases are not exhaustive. Nevertheless, the asymptotic stability of the Kalman filter established in Section 4 (see Theorem 1) is valid for all OE systems as formulated in this paper, regardless of their own stability properties.

\section{Simple analytical examples}

In order to illustrate the theoretic results presented in this paper, let us consider simple one-dimensional examples for which the convergence rates can be analytically bounded. The considered output error system is in the form of

$$
\begin{aligned}
& d x(t)=a x(t) d t \\
& d y(t)=x(t) d t+d \eta(t)
\end{aligned}
$$

with 3 different cases corresponding to $a=-1, a=0$ and $a=1$. The state transition matrix

(a scalar in this particular case) $\Phi\left(t, t_{0}\right)=e^{a\left(t-t_{0}\right)}$. The interval length in the observability Gramian is chosen as $\tau=1$ in all the cases. 
Example A1 - exponentially stable system with $a=-1$.

Straightforward computations lead to $\rho_{1}=\rho_{2}=\frac{1}{2}\left(e^{2}-1\right)$ (the symbol " $e$ " denotes Euler's number), $\alpha=\beta=1$ and, according to inequality (47),

$$
\|z(t)\|^{2} \leq \frac{2 e^{4}}{e^{2}-1} e^{-2\left(t-t_{0}\right)}\left\|z\left(t_{0}\right)\right\|^{2} .
$$

Example A2 - Lyapunov stable system with $a=0$.

In this case, $\rho_{1}=\rho_{2}=1, \gamma=1, \rho_{4}=1$ and, according to inequality (53),

$$
\|z(t)\|^{2} \leq \frac{1}{t-t_{1}}\left\|z\left(t_{1}\right)\right\|^{2}
$$

with $t_{1}=t_{0}+1$.

Example A3 - strongly unstable system with $a=1$.

In this example, $\rho_{0}=\frac{1}{2}, \rho_{1}=\rho_{2}=\frac{1}{2}\left(1-e^{-2}\right), \rho_{3}=\frac{1}{2}\left(e^{2}-1\right), \rho_{4}=\frac{1}{2}, \rho_{5}=1, \alpha=\beta=1$ and, according to inequality (70),

$$
\|z(t)\|^{2} \leq \frac{1+e^{2}}{2\left(1-e^{-2}\right)}\left(\frac{2}{1+e^{2}}\right)^{t-t_{0}}\left\|z\left(t_{0}\right)\right\|^{2} .
$$

\section{Numerical examples}

This section presents some numerical examples. The examples of LTV systems (the $A(t)$ and $\Phi\left(t, t_{0}\right)$ matrices) have been inspired by (Choi, 2010, Lecture 24). The numerical solutions are computed with the ode45 solver in Matlab.

Example N1 - exponentially stable system.

Consider

$$
A(t)=\left[\begin{array}{cc}
e^{-t}-2 & 2-e^{-t} \\
0 & e^{-t}-2
\end{array}\right], \quad C(t)=\left[\begin{array}{ll}
1 & 0
\end{array}\right], \quad R(t)=1 .
$$

The corresponding state transition matrix is

$$
\Phi\left(t, t_{0}\right)=\left[\begin{array}{cc}
\exp \left(\psi\left(t, t_{0}\right)\right) & -\psi\left(t, t_{0}\right) \exp \left(\psi\left(t, t_{0}\right)\right) \\
0 & \exp \left(\psi\left(t, t_{0}\right)\right)
\end{array}\right]
$$

with $\psi\left(t, t_{0}\right) \triangleq 2\left(t_{0}-t\right)+e^{-t_{0}}-e^{-t}$. This system is exponentially stable.

The Riccati equation (11c) is initialized with $P(0)=I_{2}$ and the homogenous system (24) with $z(0)=[1,2]^{T}$. In Figure 1 are shown the two eigenvalues (equal to the singular values) of $P(t)$, which are bounded and tend to zero. In Figure 1 is also shown the state vector $z(t)$, which converges to zero, in theory exponentially (see Theorem 2).

Example N2 - Lyapunov stable system.

Consider

$$
\begin{aligned}
& A(t)=\left[\begin{array}{cc}
\cos (0.2 t) & \sin (0.2 t) \\
-\sin (0.2 t) & \cos (0.2 t)
\end{array}\right] \\
& C(t)=\left[\begin{array}{cc}
1.5 & 0 \\
0 & 2
\end{array}\right], \quad R(t)=I_{2} \text {. }
\end{aligned}
$$



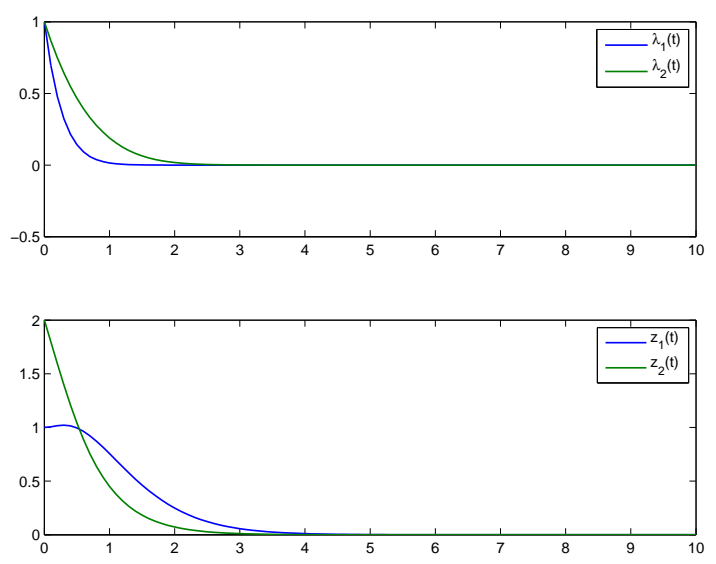

Figure 1: Example 1. Top: eigenvalues of $P(t)$, bottom: components of $z(t)$.
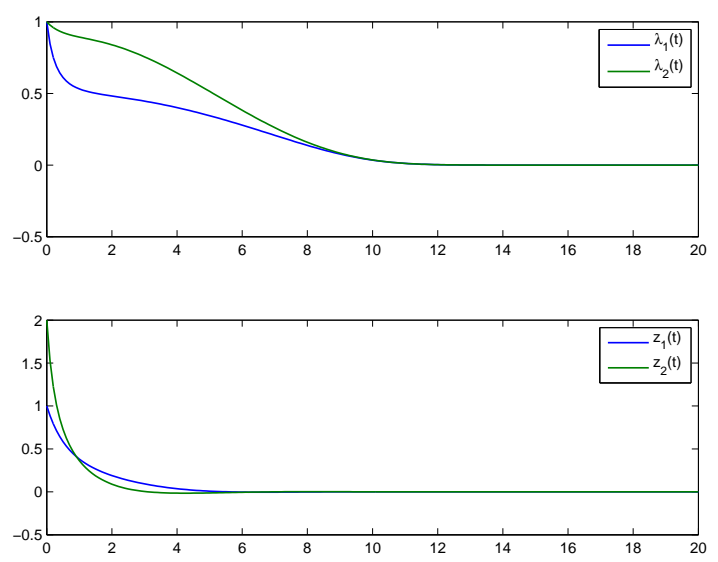

Figure 2: Example 2. Top: eigenvalues of $P(t)$, bottom: components of $z(t)$.

The corresponding state transition matrix is

$$
\Phi\left(t, t_{0}\right)=\exp \left(\varphi\left(t, t_{0}\right)\right)\left[\begin{array}{cc}
\cos \left(\psi\left(t, t_{0}\right)\right) & \sin \left(\psi\left(t, t_{0}\right)\right) \\
-\sin \left(\psi\left(t, t_{0}\right)\right) & \cos \left(\psi\left(t, t_{0}\right)\right)
\end{array}\right]
$$

with

$$
\begin{aligned}
& \varphi\left(t, t_{0}\right) \triangleq 5\left(\sin (0.2 t)-\sin \left(0.2 t_{0}\right)\right) \\
& \psi\left(t, t_{0}\right) \triangleq 5\left(\cos \left(0.2 t_{0}\right)-\cos (0.2 t)\right) .
\end{aligned}
$$

This system is Lyapunov stable.

The Riccati equation (11c) is initialized with $P(0)=I_{2}$ and the homogenous system (24) with $z(0)=[1,2]^{T}$. In Figure 2 are shown the two eigenvalues (equal to the singular values) of $P(t)$, which are bounded and tend to zero. In Figure 2 is also shown the state vector $z(t)$, which converges to zero, in theory as $1 / t$ (see Theorem 3 ).

Example N3 - strongly unstable system.

Consider

$$
A(t)=\left[\begin{array}{cc}
2-e^{-t} & e^{-t}-2 \\
0 & 2-e^{-t}
\end{array}\right], \quad C(t)=\left[\begin{array}{ll}
1 & 0
\end{array}\right], \quad R(t)=1 .
$$

The corresponding state transition matrix is

$$
\Phi\left(t, t_{0}\right)=\left[\begin{array}{cc}
\exp \left(\psi\left(t, t_{0}\right)\right) & -\psi\left(t, t_{0}\right) \exp \left(\psi\left(t, t_{0}\right)\right) \\
0 & \exp \left(\psi\left(t, t_{0}\right)\right)
\end{array}\right]
$$

with $\psi\left(t, t_{0}\right) \triangleq 2\left(t-t_{0}\right)+e^{-t}-e^{-t_{0}}$. This system is strongly unstable.

The Riccati equation (11c) is initialized with $P(0)=I_{2}$ and the homogenous system (24) with $z(0)=[1,2]^{T}$. In Figure 3 are shown the two eigenvalues (equal to the singular values) of $P(t)$, which are both upper and lower bounded. In Figure 3 is also shown the state vector $z(t)$, which converges to zero, in theory exponentially (see Theorem 4).

Example N4

Consider

$$
A(t)=\left[\begin{array}{cc}
-1+1.5 \cos ^{2} t & 1-1.5 \sin t \cos t \\
-1-1.5 \sin t \cos t & -1+1.5 \sin ^{2} t
\end{array}\right]
$$



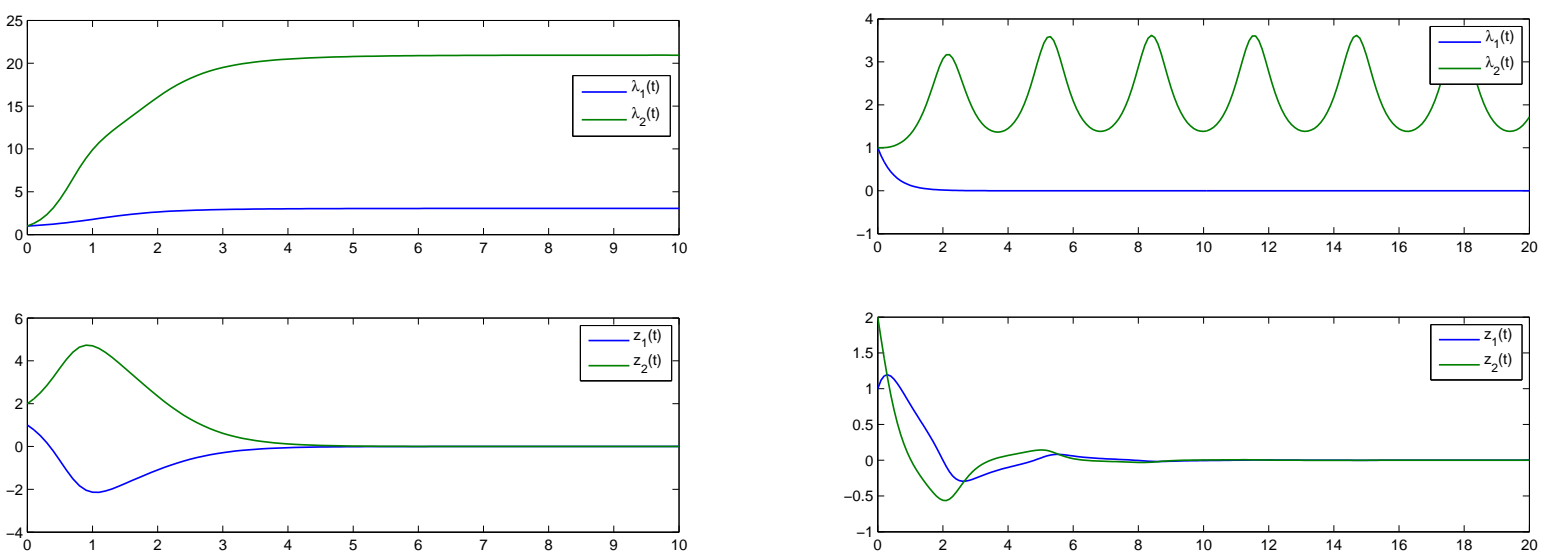

Figure 3: Example 3. Top: eigenvalues of $P(t)$, bottom: components of $z(t)$.

Figure 4: Example 4. Top: eigenvalues of $P(t)$, bottom: components of $z(t)$.

$$
C(t)=\left[\begin{array}{ll}
1 & 0
\end{array}\right], \quad R(t)=1 .
$$

The corresponding state transition matrix is

$$
\Phi(t, 0)=\left[\begin{array}{cc}
e^{0.5 t} \cos t & e^{-t} \sin t \\
-e^{0.5 t} \sin t & e^{-t} \cos t
\end{array}\right]
$$

This system is neither stable nor strongly unstable.

The Riccati equation (11c) is initialized with $P(0)=I_{2}$ and the homogenous system (24) with $z(0)=[1,2]^{T}$. In Figure 4 are shown the two eigenvalues (equal to the singular values) of $P(t)$, which are bounded. In Figure 4 is also shown the state vector $z(t)$, which converges to zero. No exponential or polynomial convergence rate is theoretically known in this case, but the result on asymptotic convergence (see Theorem 1) does apply here.

\section{Conclusion}

For any recursive algorithm running continuously in real time, the boundedness of all the involved variables is obviously an important property. It is established in this paper that, when the Kalman filter is applied to OE systems, the solution of the Riccati equation and the Kalman gain are both bounded, essentially under the observability condition. It is further shown that the Kalman filter is asymptotically stable, with exponential or polynomial convergence rate in typical situations. These results complement the classical results on the stability of the Kalman filter, which do not cover the case of OE systems.

The absence of process noise in OE systems caused a technical difficulty: unlike in the classical case, here the solution of the Riccati equation does not always have a strictly positive definite lower bound, therefore the associated "natural" Lyapunov function cannot be used in the usual sense in the convergence proofs. Despite this difficulty, the results of this paper show that, the essential condition ensuring the stability of the Kalman filter for OE systems is the uniform complete observability.

The Kalman filter is optimal in the sense of minimal error covariance. Reducing process and output noises generally leads to smaller error covariance, including the case of zeroing the process noise. However, the reduction of error covariance may not be in favor of the average error convergence, in particular when the process noise is totally absent. In some cases considered in 
this paper, exponential convergence is not guaranteed, but not explicitly excluded either. This point still needs investigations in future studies.

\section{Appendix - Proof of Lemma 1}

First prove $\|M\| \leq \xi \Rightarrow\|M v\| \leq \xi\|v\|$.

By basic properties of the matrix spectral norm, suppose $\|M\| \leq \xi$, then

$$
\|M v\| \leq\|M\|\|v\| \leq \xi\|v\| .
$$

Now let us prove the converse.

Suppose $\|M v\| \leq \xi\|v\|$ for all $v \in \mathbb{R}^{n}$, then by the definition of the matrix spectral norm,

$$
\begin{aligned}
\|M\| & =\max _{\|v\|=1}\|M v\| \\
& \leq \max _{\|v\|=1} \xi\|v\| \\
& =\xi .
\end{aligned}
$$

\section{References}

Anderson, B. D., , Bitmead, R. R., Johnson, C. R. J., Kokotovic, P. V., Kosut, R. L., Mareels, I. M., Praly, L., Riedle, B. D., 1986. Stability of adaptive systems: passivity and averaging analysis. Signal processing, optimization, and control. MIT Press, Cambridge, Massachusetts.

Anderson, B. D. O., Moore, J. B., 1979. Optimal Filtering. Prentice-Hall, Inc., New Jersey.

Arnold, W. F., Laub, A. J., 1984. Generalized eigenproblem algorithms and software for algebraic Riccati equations. Proceedings of the IEEE 72 (12), 1746-1754.

Choi, J., 2010. Linear systems and controls. Lecture notes, http://www.egr.msu.edu/classes/me851/jchoi /lecture/index.html.

Delyon, B., 2001. A note on uniform observability. IEEE Transactions on Automatic Control 46 (8), $1326-1327$.

Forssell, U., Ljung, L., 2000. Identification of unstable systems using output error and box-jenkins model structures. IEEE Transactions on Automatic Control 45 (1), 137-141.

Goodman, D. M., Dudley, D. G., 1987. An output error model and algorithm for electromegnetic system identification. Circuits Systems Signal Process 6 (4), 471-505.

Grewal, M. S., Andrews, A. P., 2015. Kalman Filtering: Theory and Practice with Matlab, 4th Edition. Wiley-IEEE Press.

Ioannou, P., Sun, J., 1996. Robust Adaptive Control. Prentice Hall, electronic copy http://wwwrcf.usc.edu/ ioannou/Robust_Adaptive_Control.htm.

Jazwinski, A. H., 1970. Stochastic Processes and Filtering Theory. Academic Press, New York and London.

Kalman, R. E., 1963. New methods in Wiener filtering theory. In: Bogdanoff, J. L., Kozin, F. (Eds.), Proceedings of the First Symposium on Engineering Applications of Random Function Theory and Probability. John Wiley \& Sons, New York, pp. 270-388. 
Kim, P., 2011. Kalman Filter for Beginners: with Matlab Examples. CreateSpace.

Laub, A. J., 1979. A Schur method for solving algebraic Riccati equations. IEEE Transactions on Automatic Control AC-24 (6), 913-921.

Ljung, L., 1999. System Identification: Theory for the User, 2nd Edition. PTR Prentice Hall, Upper Saddle River, NJ, USA.

Ni, B., Zhang, Q., July 2013. Kalman filter for process noise free systems. In: IFAC International Workshop on Adaptation and Learning in Control and Signal Processing (ALCOSP). Caen, France.

Ni, B., Zhang, Q., 2015. Stability of the Kalman filter for output error systems. In: Submitted to the 17th IFAC Symposium on System Identification. Beijing.

Pengov, M., Richard, E., Vivalda, J.-C., 2001. On the boundedness of the solutions of the continuous Riccati equation. Journal of Inequalities and Applications 6 (6), 641-649.

Rugh, W. J., 1995. Linear System Theory. Prentice Hall.

Sastry, S., Bodson, M., 1989. Adaptive Control: Stability, Convergence and Robustness. Prentice-Hall.

Wang, L., Ortega, R., Su, H., Liu, Z., Liu, X., 2015. A robust output error identifier for continuous-time systems. International Journal of Adaptive Control and Signal Processing 29 (4), 443-456.

Zarchan, P., Musof, H., 2005. Fundamentals of Kalman filtering: a practical approach. AIAA.

Zhang, L., Zhang, Q., 2015. Observability conservation by output feedback and observability Gramian bounds. Automatica 60, 38-42.

URL https://hal.inria.fr/hal-01231786 